Check for updates

Cite this: J. Mater. Chem. A, 2019, 7, 3206

Received 1st October 2018

Accepted 14th January 2019

DOI: 10.1039/c8ta09501a

rsc.li/materials-a

\section{Charge carrier dynamics in tantalum oxide overlayered and tantalum doped hematite photoanodes $\dagger$}

\author{
Tero-Petri Ruoko, (D) $t^{* a}$ Arto Hiltunen, (D) a Tomi livonen, (D) ${ }^{b}$ Riina Ulkuniemi, ${ }^{c}$ \\ Kimmo Lahtonen, (D) ' Harri Ali-Löytty, ID c Kenichiro Mizohata, (iD d Mika Valden, ${ }^{c}$ \\ Markku Leskeläb and Nikolai V. Tkachenko (D)
}

\begin{abstract}
We employ atomic layer deposition to prepare $50 \mathrm{~nm}$ thick hematite photoanodes followed by passivating them with a $0.5 \mathrm{~nm}$ thick $\mathrm{Ta}_{2} \mathrm{O}_{5}$-overlayer and compare them with samples uniformly doped with the same amount of tantalum. We observe a three-fold improvement in photocurrent with the same onset voltage using Ta-overlayer hematite photoanodes, while electrochemical impedance spectroscopy under visible light irradiation shows a decreased amount of surface states under water splitting conditions. The Tadoped samples have an even higher increase in photocurrent along with a $0.15 \mathrm{~V}$ cathodic shift in the onset voltage and decreased resistivity. However, the surface state capacitance for the Ta-doped sample is twice that of the reference photoanode, which implies a larger amount of surface hole accumulation. We further utilize transient absorption spectroscopy in the sub-millisecond to second timescale under operating conditions to show that electron trapping in both $\mathrm{Ta}_{2} \mathrm{O}_{5}$-passivated and $\mathrm{Ta}$-doped samples is markedly reduced. Ultrafast transient absorption spectroscopy in the sub-picosecond to nanosecond timescale shows faster charge carrier dynamics and reduced recombination in the Ta-doped hematite photoanode resulting in the increased photoelectrochemical performance when compared with the $\mathrm{Ta}_{2} \mathrm{O}_{5}$-overlayer sample. Our results show that passivation does not affect the poor charge carrier dynamics intrinsic to hematite based photoanodes. The Ta-doping strategy results in more efficient electron extraction, solving the electron trapping issue and leading to increased performance over the surface passivation strategy.
\end{abstract}

\section{Introduction}

Photoelectrochemical water splitting with stable metal oxide materials produces environmentally friendly hydrogen gas that can be transported over large distances to be burned in conventional gas power plants or converted directly into electricity using fuel cells. This solution allows to store solar energy directly into chemical bonds, thus potentially solving the

${ }^{a}$ Chemistry and Advanced Materials, Laboratory of Chemistry and Bioengineering, Tampere University of Technology, P.O. Box 541, FI-33101 Tampere, Finland. E-mail: tero-petri.ruoko@liu.se

${ }^{b}$ Department of Chemistry, University of Helsinki, P.O. Box 55, FI-00014 Helsinki, Finland

'Surface Science Group, Laboratory of Photonics, Tampere University of Technology, P.O. Box 692, FI-33101 Tampere, Finland

${ }^{d}$ Department of Physics, University of Helsinki, P.O. Box 43, FI-00014 Helsinki, Finland $\dagger$ Electronic supplementary information (ESI) available: XPS elemental analysis table and transient absorption fitting details, decay component spectra, and additional decays. See DOI: 10.1039/c8ta09501a

\$ Present address: Laboratory of Organic Electronics, Department of Science and Technology, Linköping University, SE-60174 Norrköping, Sweden. problems associated with intermittent and localized solar electricity production.

Hematite $\left(\alpha-\mathrm{Fe}_{2} \mathrm{O}_{3}\right)$ has been regarded as one of the most promising candidates for photoelectrochemical water splitting ever since it was first used for this purpose by Hardee and Bard in 1976. ${ }^{1}$ Hematite has numerous advantages as a photoanode material, such as a near optimal band gap of $2.2 \mathrm{eV}$ that results in a theoretical solar-to-hydrogen efficiency of $12.9 \%,{ }^{2}$ high chemical stability in non-acidic conditions, natural abundance, and low production costs. ${ }^{3,4}$ However, the efficiencies of hematite photoanodes remain low due to a few intrinsic drawbacks: (i) a flat band potential that is too low for water reduction, (ii) poor electron mobility, and (iii) a small hole diffusion length of 2-20 nm. ${ }^{5-8}$ Furthermore, surface states at the hematite-electrolyte interface can also act as recombination centers that drastically decrease the photoanode performance. ${ }^{9}$

Metal oxide surfaces are spontaneously hydroxylated in aqueous conditions. ${ }^{10}$ Iandolo et al. ${ }^{11}$ showed that hematite surfaces are populated by $\mathrm{OH}$ - and O-terminations of the iron oxide lattice, both of which act as surface states. The hydroxylterminated surface states are occupied mid-gap states that act as surface recombination centers at low applied bias voltages. 
On the other hand, the oxo-terminated states are located energetically on the top of the valence band, and take part in the oxygen evolution reaction (OER) at medium biases. Passivation of the hematite surface states has been shown to increase the performance of hematite photoanodes. ${ }^{4}$ Numerous different methods have been employed to passivate hematite surfaces, including high temperature annealing, ${ }^{\mathbf{1 2}}$ acid treatment, ${ }^{\mathbf{1 3}}$ deposition of possibly passivating catalysts such as cobalt phosphate (Co-Pi), $\mathrm{IrO}_{x}$ or $\mathrm{NiFeO}_{x},{ }^{14-19}$ and depositing thin metal oxide overlayers, such as $\mathrm{TiO}_{2},{ }^{20-22} \mathrm{Ga}_{2} \mathrm{O}_{3},{ }^{14,17}$ or $\mathrm{Al}_{2} \mathrm{O}_{3} \cdot{ }^{14,17-19,23-25}$ Out of the metal oxide passivating layers used for hematite photoanodes, $\mathrm{TiO}_{2}$ has yielded inconclusive results, varying from no improvement ${ }^{\mathbf{2 0 , 2 3}}$ to improvement only after high temperature annealing ${ }^{22}$ or a solution deposition process. ${ }^{26}$ On the other hand, $\mathrm{Ga}_{2} \mathrm{O}_{3}$ (ref. 14) and to some extent also $\mathrm{Al}_{2} \mathrm{O}_{3}$ (ref. 27) are susceptible to dissolution in basic electrolytes. For these reasons we chose to study the effects of $\mathrm{Ta}_{2} \mathrm{O}_{5}$ as an overlayer and dopant in hematite photoanodes.

$\mathrm{Ta}_{2} \mathrm{O}_{5}$ is a large band gap $(3.9 \mathrm{eV})^{28}$ metal oxide which is often used in anti-reflective coatings for solar cells and sensors. Due to its high-permittivity and excellent chemical stability it has also found applications in the semiconductor industry, such as in dielectric layers for transistors and storage capacitors. ${ }^{29}$ The band levels of $\mathrm{Ta}_{2} \mathrm{O}_{5}$ straddle the water oxidation and reduction potentials, ${ }^{28}$ making it a potential candidate for use as an overall water splitting photocatalyst. ${ }^{30}$ However, due to the large band gap it cannot be excited with visible light irradiation, greatly limiting the obtainable photocurrent. $\mathrm{Ta}_{2} \mathrm{O}_{5}$ has previously been used as a dopant in hematite photoanodes, but the results have been inconclusive. Ta-doping increases the conductivity of hematite, ${ }^{31}$ but photoelectrochemical improvements due to the doping have varied from non-existent, ${ }^{32}$ to a 3.5 time improvement for water oxidation, ${ }^{33}$ reaching all the way to a 32 time improvement in photocurrent over pristine hematite in the presence of scavengers. ${ }^{34} \mathrm{Ta}_{2} \mathrm{O}_{5}$ was recently used as a surface passivation layer for $\mathrm{ZnO}$ photoanodes, leading to nearly two-fold improved photoelectrochemical performance. ${ }^{35}$ The chemical stability of $\mathrm{Ta}_{2} \mathrm{O}_{5}$ promises more stable passivating layers in non-neutral solutions than $\mathrm{Ga}_{2} \mathrm{O}_{3}$ or $\mathrm{Al}_{2} \mathrm{O}_{3}$. $\mathrm{Ta}_{2} \mathrm{O}_{5}$ was recently utilized as an overlayer for hematite photoanodes, which resulted in approximately a three-fold increase in the photocurrent over pristine nanostructured hematite photoanodes. ${ }^{36}$

Herein, we utilized $50 \mathrm{~nm}$ thick hematite photoanodes made by atomic layer deposition (ALD). The deposition method and sample thickness were chosen to have a uniform sample surface, while keeping the sample absorbance relatively low in order to characterize the charge carrier dynamics using transient absorption spectroscopy (TAS). The $\mathrm{Ta}_{2} \mathrm{O}_{5}$-passivated samples were prepared by depositing an ultrathin (roughly 0.5 $\mathrm{nm}$ ) overlayer by ALD, whereas Ta-doped samples were prepared by applying one $\mathrm{Ta}_{2} \mathrm{O}_{5}$ deposition cycle once every hundred hematite deposition cycles. This resulted in the same number of Ta-precursor cycles for both overlayer and doped samples. We observed a three-fold improvement in photocurrent with the same onset voltage using $\mathrm{Ta}_{2} \mathrm{O}_{5}$-overlayer hematite photoanodes. Electrochemical impedance spectroscopy (EIS) under visible light irradiation showed a decreased amount of surface states under water splitting conditions, indicating that the surface has indeed been passivated. However, the Ta-doped samples showed an even larger increase in photocurrent along with a $0.15 \mathrm{~V}$ cathodic shift in the onset voltage and decreased resistivity. Remarkably, the surface state capacitance for the Tadoped hematite was two times larger than for the reference photoanode. We utilized TAS in the sub-millisecond to second timescale under water splitting conditions to show that electron trapping in both $\mathrm{Ta}_{2} \mathrm{O}_{5}$-passivated and Ta-doped samples was markedly reduced. Ultrafast TAS in the sub-picosecond to nanosecond timescale indicated faster charge carrier dynamics in the Ta-doped hematite photoanode to be a probable reason for the increased photoelectrochemical performance.

\section{Experimental}

Hematite photoanodes were grown on fluorine doped tin oxide (FTO) coated glass substrates (Sigma-Aldrich TEC7, $\sim 7 \Omega \mathrm{sq}^{-1}$ ) using the $\mathrm{FeCl}_{3}+\mathrm{H}_{2} \mathrm{O}$ deposition process $^{37}$ in a $\mathrm{F}-120$ ALD reactor (ASM Microchemistry) operated in the cross-flow configuration. ${ }^{38}$ The reference and overlayer hematite samples were grown at $400{ }^{\circ} \mathrm{C}$ using 1000 ALD-cycles, after which the samples were annealed at $600{ }^{\circ} \mathrm{C}$ for 60 minutes in air. The resulting layer thickness was $50 \mathrm{~nm}$. The $\mathrm{Ta}_{2} \mathrm{O}_{5}$-overlayer was grown by applying 10 cycles of the $\mathrm{Ta}\left(\mathrm{OC}_{2} \mathrm{H}_{5}\right)_{5}+\mathrm{H}_{2} \mathrm{O}$ deposition process at $300{ }^{\circ} \mathrm{C}$ on the annealed hematite surface. ${ }^{39}$ The deposition temperature of the overlayer was lower in order to avoid the thermal decomposition of $\mathrm{Ta}\left(\mathrm{OC}_{2} \mathrm{H}_{5}\right)_{5}$, which becomes significant at temperatures above $325{ }^{\circ} \mathrm{C}$. The resulting overlayer thickness was $0.5 \mathrm{~nm}$. The Ta-doped samples were grown by repeating the $\mathrm{Ta}\left(\mathrm{OC}_{2} \mathrm{H}_{5}\right)_{5}+\mathrm{H}_{2} \mathrm{O}$ cycle once after every $99 \mathrm{FeCl}_{3}+\mathrm{H}_{2} \mathrm{O}$ cycles at $300{ }^{\circ} \mathrm{C}$ for a total of 1000 cycles, followed by annealing at $600{ }^{\circ} \mathrm{C}$ for 60 minutes in air. All asprepared samples were finally annealed at $300{ }^{\circ} \mathrm{C}$ for 60 minutes in air to remove possible organic contaminants. The ALD deposition process is presented schematically in Fig. 1. Note that Fig. 1 does not represent the final sample structure due to the rough FTO substrate and the annealing steps inducing diffusion of tantalum within the samples.

X-ray photoelectron spectroscopy (XPS) measurements were performed utilizing a non-monochromatized $\mathrm{Al} \mathrm{K \alpha} \mathrm{X}$-ray source $(h \nu=1486.6 \mathrm{eV})$ and an Argus hemispherical electron spectrometer (Omicron Nanotechnology $\mathrm{GmbH}$ ) installed in

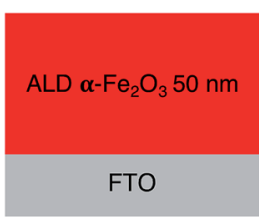

Reference

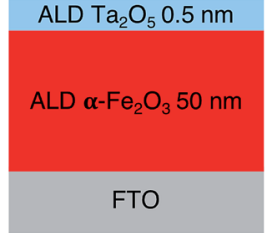

$\mathrm{Ta}_{2} \mathrm{O}_{5}$-overlayer
ALD $\alpha-\mathrm{Fe}_{2} \mathrm{O}_{3} 50 \mathrm{~nm}$ ALD $\mathrm{Ta}_{2} \mathrm{O}_{5} 1: 100$ cycles

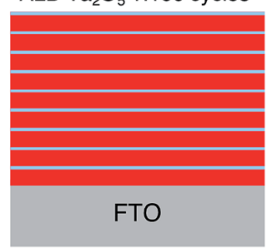

Ta-doped
Fig. 1 Schematic representation of the ALD deposition processes for the reference, $\mathrm{Ta}_{2} \mathrm{O}_{5}$-overlayer, and Ta-doped hematite photoanodes. 
a multifunctional UHV system with a base pressure below $1 \times$ $10^{-10}$ mbar. $^{40}$ The binding energy scale of the XPS spectrometer was calibrated according to the metallic $\mathrm{Cu} 2 \mathrm{p}_{3 / 2}$ at $932.40 \mathrm{eV}$ and $\mathrm{Ag} 3 \mathrm{~d}_{5 / 2}$ at $368.00 \mathrm{eV}$. The surface elemental concentrations and chemical states of compounds were identified by analyzing high-resolution XPS spectra using CasaXPS software (Version 2.3.18 PR 1.0). After a Shirley-type background subtraction, the peaks were least-squares fitted with a combination of symmetric Gaussian-Lorentzian line shapes. The relative atomic concentrations were calculated using Scofield photoionization cross sections ${ }^{41}$ and the experimentally measured transmission function of the Argus analyser.

Depth profiles for the hematite photoanodes were calculated using combined data from time-of-flight elastic recoil detection analysis (ToF-ERDA) and Rutherford backscattering spectroscopy (RBS) measurements. The ion beam measurements were done at the Accelerator Laboratory at University of Helsinki using a 5 MV tandem accelerator. ${ }^{42}$ For the ToF-ERDA measurements, a $50 \mathrm{MeV}{ }^{127} \mathrm{I}^{9+}$ ion beam was used whereas the RBS measurements were done using a $8 \mathrm{MeV}^{12} \mathrm{C}^{+}$ion beam.

Field emission scanning electron microscopy images were taken with a Carl Zeiss Ultra 55 with an acceleration voltage of 4 $\mathrm{kV}$. The absorption spectra of the hematite photoanodes were measured with a Shimadzu UV-3600 UV-Vis-NIR spectrophotometer in transmission mode.

All measurements with bias voltages were carried out in a three electrode photoelectrochemical cell (PECC-2, Zahner) in $0.1 \mathrm{M} \mathrm{NaOH}(\mathrm{pH} \approx 12.8)$. The bias voltages were set using a potentiostat (CompactStat, Ivium Technologies) or a two channel source-monitoring unit (E5272A, Agilent Technologies) using a $\mathrm{Ag} / \mathrm{AgCl}$ reference electrode (RE-5B, BASi, $3 \mathrm{M} \mathrm{NaCl}$ ) and a platinum wire counter electrode. Electrochemical impedance measurements were scanned in the frequency range of $10 \mathrm{kHz}$ to $10 \mathrm{mHz}$ with a $10 \mathrm{mV}$ AC signal. All voltages are reported relative to the reversible hydrogen electrode (RHE) scale, calculated using $E_{\mathrm{RHE}}=E^{\circ}+E_{\mathrm{Ag} / \mathrm{AgCl}}+0.059 \mathrm{pH}$, where $E^{\circ}$ is the reference potential of the $\mathrm{Ag} / \mathrm{AgCl}$ electrode $\left(0.206 \mathrm{~V}_{\mathrm{SHE}}\right.$ at 25 $\left.{ }^{\circ} \mathrm{C}\right), E_{\mathrm{Ag} / \mathrm{AgCl}}$ is the measured potential versus the $\mathrm{Ag} / \mathrm{AgCl}$ electrode. Current density-voltage and electrochemical impedance measurements were performed under simulated sunlight obtained with a class AAA solar simulator (Sciencetech SS150-AAA) adjusted to one sun total intensity with a calibrated $\mathrm{Si}$ reference cell (Oriel 91150V) with electrolyte-side illumination.

Transient absorption in the sub-ms to $\mathrm{s}$ timescales was studied using the flash-photolysis method in transmission mode. The third harmonic (F015, Solar TII, $355 \mathrm{~nm}, 0.2 \mathrm{~mJ}$ $\mathrm{cm}^{-2}$, electrolyte-side illumination) of a Nd:YAG laser (LF117, Solar TII, $10 \mathrm{~ns}$ pulse width, $0.25 \mathrm{~Hz}$ repetition rate) was used as the excitation pump. A stabilized halogen light source (SLS201/ M, Thorlabs, $9 \mathrm{~W}$ ) was used as the probe beam. The monochromatic (CM110, Digikröm) transient signal was detected with a silicon photoreceiver (2051-FS, New Focus) and recorded with a digitizing oscilloscope (TDS5032B, Tektronix). An in-lab program was used to manage the experiment. The transient decays were averaged over 200-1000 laser shots.

Transient absorption in the sub-ps to ns timescales was studied using the pump-probe method in transmission mode.
The fundamental laser pulses were generated with a Ti:Sapphire laser (Libra F, Coherent Inc., $800 \mathrm{~nm}, 100$ fs pulse width, repetition rate $1 \mathrm{kHz}$ ). $90 \%$ of the fundamental beam energy was directed to an optical parametric amplifier (Topas C, Light Conversion Ltd.) to produce the desired wavelength excitation pump pulses (355 $\mathrm{nm}$, approximately $0.5 \mathrm{~mm}$ diameter at the sample, attenuated to $0.3 \mathrm{~mJ} \mathrm{~cm}^{-2}$ with neutral density filters). Roughly $10 \%$ of the fundamental laser beam energy was directed through a motorized translational stage (delay line) to a $1 \mathrm{~mm}$ thick water cuvette for white continuum generation of probe pulses. The probe light was split into the reference and signal beams. The measurement system (ExciPro, CDP Inc.) was equipped with a Si CCD pair for monitoring the transient absorption in the visible range. A chopper synchronized with the fundamental laser pulses was used to block every second pump pulse, and the absorbance change was calculated from consecutive pulses. The spectra were averaged over 10000 excitation pulses for each delay time.

\section{Results and discussion}

The ALD-grown hematite creates a uniform layer on the rough FTO substrate, as shown in the scanning electron microscope (SEM) images of the photoanode surfaces in Fig. 2. The deposition of a $0.5 \mathrm{~nm} \mathrm{Ta}_{2} \mathrm{O}_{5}$ overlayer does not change the surface morphology of the photoanode. However, the introduction of the $\mathrm{Ta}_{2} \mathrm{O}_{5}$ precursor during hematite film growth as a dopant leads to the formation of a rougher surface texture with larger crystallites of up to $500 \mathrm{~nm}$ in diameter. A disturbance of hematite nanorod growth upon Ta-doping, ${ }^{33}$ as well as a preferential crystal growth along the $\{001\}$ facet have been previously reported. ${ }^{34}$ Thus, we conclude that the Ta-dopant disturbs the uniform hematite growth, leading to the formation of larger crystallites.

X-ray photoelectron spectroscopy (XPS) was employed for the analysis of Ta addition on the chemical composition of the hematite surfaces. The relative surface composition of elements and chemical species is presented in Table S1. $\dagger$ Fig. 3 depicts the Fe $2 \mathrm{p}$ and Ta $4 \mathrm{f}$ spectra of the samples (spectra with fitted components are presented in Fig. S1†). The spectral features of Fe 2p were similar for all samples, and the binding energies of the Fe $2 \mathrm{p}_{3 / 2}$ peak at $710.4 \mathrm{eV}$ and the satellite peak at $719.5 \mathrm{eV}$ are characteristic to the oxidation state of $\mathrm{Fe}^{3+}$ in, for example, hematite $\left(\alpha-\mathrm{Fe}_{2} \mathrm{O}_{3}\right){ }^{43}$ For the Ta-doped and $\mathrm{Ta}_{2} \mathrm{O}_{5}$-overlayer hematite samples the binding energy of $\mathrm{Ta} 4 \mathrm{f}_{7 / 2}$ at $25.5 \mathrm{eV}$ can be attributed to $\mathrm{Ta}^{5+}$ in accordance with the value observed by Zhang et al. ${ }^{34}$ Neither Fe nor Ta species were observed at lower oxidation states than $3+$ or $5+$, respectively, which indicates a low amount of oxygen vacancies. No change in the Ta $4 \mathrm{f}$ peak position was observed despite the change in the $\mathrm{Ta} / \mathrm{Fe}$ ratio of the Ta-doped and $\mathrm{Ta}_{2} \mathrm{O}_{5}$-overlayer hematite samples from 0.16 to 0.58 , respectively. The high $\mathrm{Ta} / \mathrm{Fe}$ ratio of the Ta-doped sample compared to the bulk ratio (0.01) indicates annealing induced surface segregation of tantalum and the formation of a Ta diffusion profile through the sample, however, the even higher ratio in the $\mathrm{Ta}_{2} \mathrm{O}_{5}$-overlayer hematite suggests the formation of a surface layer. 

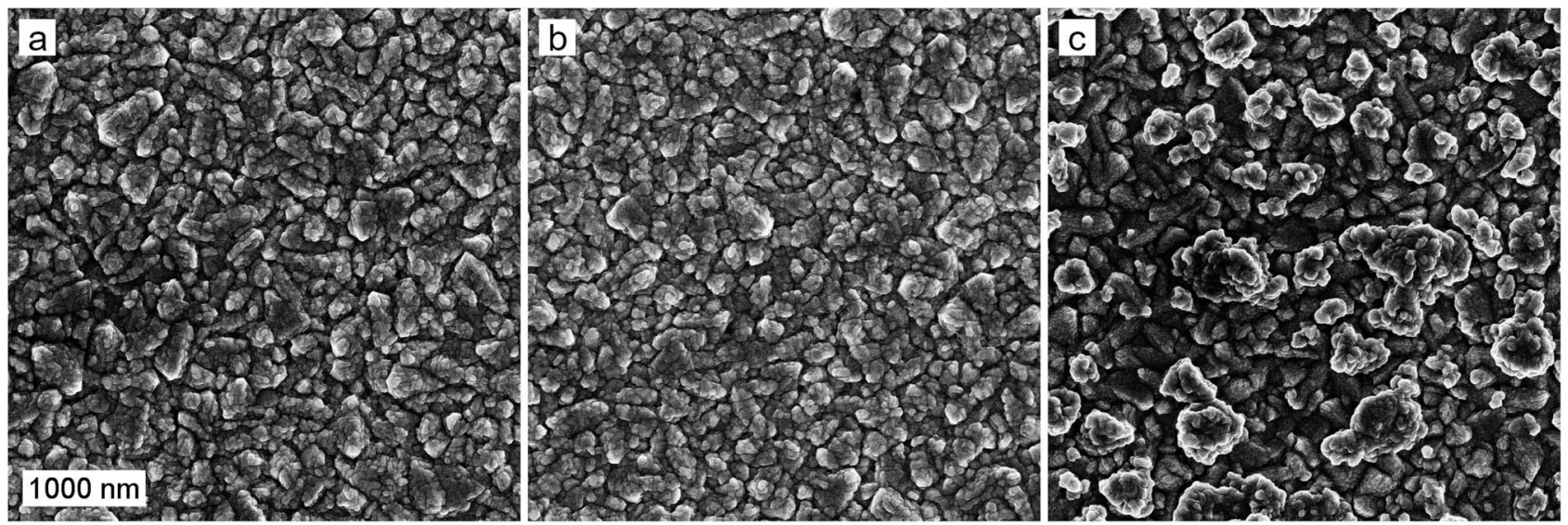

Fig. 2 SEM micrographs of the (a) reference, (b) $\mathrm{Ta}_{2} \mathrm{O}_{5}$-overlayer, and (c) Ta-doped hematite photoanodes. The scale bar represents $1000 \mathrm{~nm}$.

a

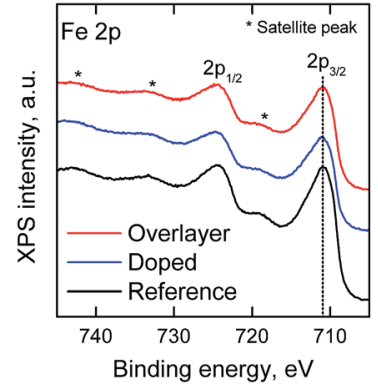

b

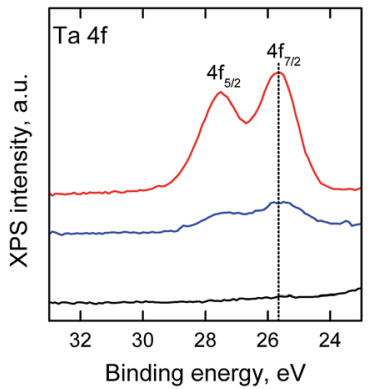

Fig. 3 (a) Fe 2p and (b) Ta 4 XPS spectra of the hematite photoanodes.

We determined the elemental depth distribution within the hematite photoanodes using time-of-flight elastic recoil detection analysis (ToF-ERDA) and Rutherford backscattering spectroscopy (RBS). The elemental depth profiles and the tantalum distribution for the hematite photoanodes are presented in Fig. 4 and S2. $\dagger$

Diffusion of tin from the FTO substrate towards the surface can be seen for all samples in Fig. S2, $\uparrow$ however, tin does not diffuse all the way to the surface. The Tof-ERDA results show very little carbon within the films, indicating that the carbon that was observed in the XPS measurements is a surface contaminant from the atmosphere. Tantalum is not observed for the reference photoanode. The $\mathrm{Ta}_{2} \mathrm{O}_{5}$-overlayer hematite has
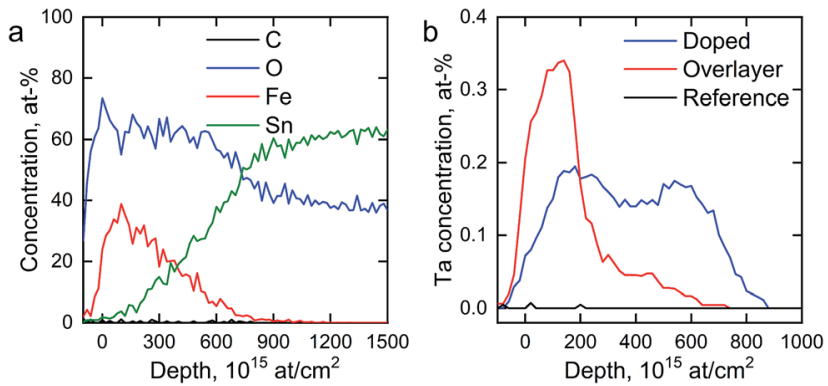

Fig. 4 (a) ToF-ERDA elemental depth profile of the reference photoanode and (b) Ta distribution in the hematite photoanodes.

a very narrow distribution of tantalum with an extended tail, showing that the $\mathrm{Ta}_{2} \mathrm{O}_{5}$ deposition forms a clearly defined overlayer with tantalum diffusing slightly into the hematite layer in the post-deposition annealing. On the other hand, the tantalum distribution in the Ta-doped hematite photoanode is more uniform, suggesting that the annealing step has dispersed the tantalum throughout the hematite layer. A slight surface enrichment can also be seen in the Ta-doped hematite photoanode, as was suggested by the XPS results.

The absorption spectra of the reference and $\mathrm{Ta}_{2} \mathrm{O}_{5}$-overlayer hematite presented in Fig. 5a are almost identical, supporting the fact that the hematite layer thickness is identical for both films. $\mathrm{Ta}_{2} \mathrm{O}_{5}$ has a band gap of $3.9 \mathrm{eV},{ }^{28}$ leading to an overlapping absorption with the glass substrate at around $315 \mathrm{~nm}$. The increase in absorption for the doped sample is a result of the rougher surface of the photoanode scattering incident light, leading to a seeming increase in absorption already at $800 \mathrm{~nm}$. However, the Ta-doped photoanode absorbance is roughly the same as for the other photoanodes when the scattering is taken into consideration.

Current density-voltage curves under 1 sun AM1.5G illumination are presented in Fig. 5b. The reference hematite films give a photoresponse similar to what has been previously reported for ALD-grown hematite films of similar thickness, ${ }^{20}$ allowing us to conclude that the photoanodes work as expected. Passivating the surface with 10 cycles $(\sim 0.5 \mathrm{~nm})$ of $\mathrm{Ta}_{2} \mathrm{O}_{5}$ results
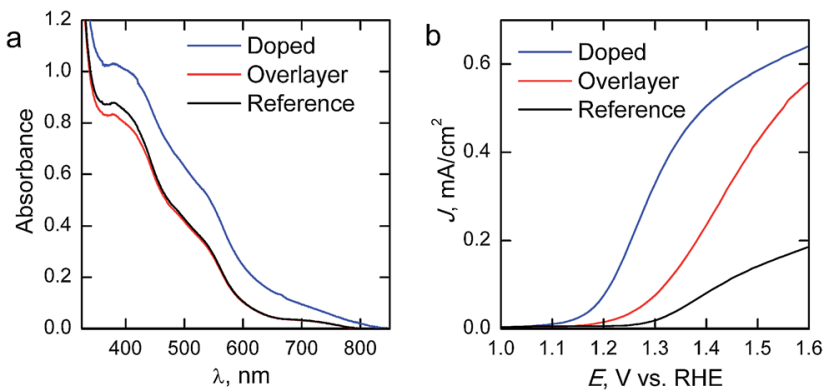

Fig. 5 (a) Absorption spectra and (b) photocurrent density-voltage curves of the hematite photoanodes under 1 sun AM1.5G illumination. 
in a three-fold improvement on the photocurrent over the bare hematite photoanode in the whole potential range, while no changes are observed in the onset potential. In a recent paper Forster et $a .^{36}$ concluded that the optimum $\mathrm{Ta}_{2} \mathrm{O}_{5}$ overlayer thickness lies between 0.5 and $2 \mathrm{~nm}$, which also resulted in approximately a three-fold improvement on the photocurrent of nanostructured hematite photoanodes. The improvements in photocurrent are attributed to a passivation of surface states that act as recombination centers. However, our results show that Ta-doping with the same number of ALD-cycles leads to an even larger improvement in photoelectrochemical performance. The Ta-doping approach results in a larger increase in the photocurrent than the $\mathrm{Ta}_{2} \mathrm{O}_{5}$-overlayer, along with a $0.15 \mathrm{~V}$ cathodic shift in the onset potential, leading to significantly higher photocurrents in the whole potential range shown. Furthermore, the slope of the photocurrent rise after the onset potential is steeper for the Ta-doped sample, which indicates lower resistivity. The decreased resistivity is presumably due to the Ta-doping inducing preferential growth along the $\{001\}$ facet of hematite, which has previously been reported to lead up to a one order of magnitude increase in conductivity. ${ }^{34}$

We performed electrochemical impedance spectroscopy (EIS) measurements in order to characterize the surface states present in our materials. Representative Nyquist plots of the materials held at $1.40 \mathrm{~V}$ vs. RHE under 1 sun AM1.5G illumination are presented in Fig. $6 \mathrm{a}$. The reference hematite exhibits two clear semicircles, which indicates the presence of two capacitances in the system. The higher frequency (left side) semicircle is only weakly bias dependent, whereas the lower frequency (right side) semicircle first grows with increased bias voltages before disappearing at higher potentials. For the $\mathrm{Ta}_{2} \mathrm{O}_{5}$-overlayer hematite two semicircles are also observed, but both semicircles have a much smaller diameter than for the reference hematite. On the other hand, at this applied potential the Ta-doped hematite only exhibits one semicircle, which almost exactly matches with the high frequency semicircle observed for the reference hematite film. However, two clear semicircles are observed for this sample at lower applied potentials.

To fit the EIS measurements we used the equivalent circuit that has been recently employed for hematite photoanodes by the groups of Hamann and Bisquert, shown in Fig. 6b.9,25,44,45 The equivalent circuit is composed of five elements: the series
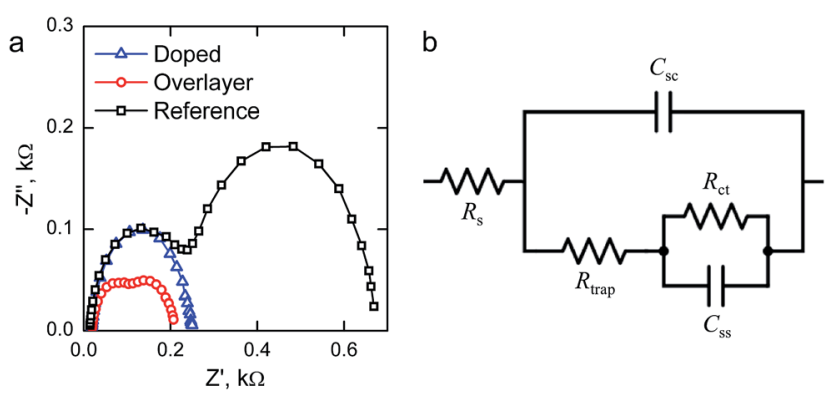

Fig. 6 (a) Representative Nyquist plots of the hematite photoanodes determined under 1 sun AM1.5G illumination at $1.40 \mathrm{~V}$ vs. RHE and (b) the equivalent circuit used to fit the measured impedance spectra. resistance between the FTO and hematite $R_{\mathrm{s}}$, the space-charge region capacitance of bulk hematite $C_{\mathrm{sc}}$, the charge trapping resistance to surface states $R_{\text {trap }}$, the surface state chemical capacitance $C_{\mathrm{ss}}$, and the charge transfer resistance from the surface states to solution $R_{\text {ct. }}{ }^{\mathbf{9 , 1 1 , 2 5}}$

The surface state capacitance $\left(C_{\mathrm{ss}}\right)$ and charge transfer resistance $\left(R_{\mathrm{ct}}\right)$ of the hematite photoanodes are presented in Fig. 7 as a function of applied bias voltage. The $C_{\mathrm{ss}}$ has a nearly Gaussian dependence on the bias voltage just above the onset potential, indicating that water oxidation occurs through surface states and via direct hole transfer from the valence band at moderate and highly anodic bias voltages, respectively. ${ }^{\mathbf{9 1 1}} C_{\mathrm{sS}}$ begins to rise at the same voltage as the photocurrent onset, and reaches a maximum at the potential at which the photocurrent rises most steeply, indicative of capacitive charging of the photoanode surface. ${ }^{45}$ The $\mathrm{Ta}_{2} \mathrm{O}_{5}$-overlayer hematite has a lower surface state capacitance than the reference hematite while the photocurrent is three times larger, indicating that the surface has been efficiently passivated. On the other hand, the $C_{\mathrm{ss}}$ of the Ta-doped hematite photoanode is more than two times larger than for the reference hematite, even though it exhibits the highest photocurrent values. This is a clear indication that the Ta-doping has not passivated the surface, yet it performs with the highest efficiency. The $R_{\text {ct }}$ of all of the photoanodes decreases concomitantly with the increase in $C_{\mathrm{ss}}$ until the surface state capacitance drops due to hole transfer from the valence band becoming prevalent. The $R_{\mathrm{ct}}$ for the Ta-doped hematite is an order of magnitude lower at all bias voltages where the surface state capacitance is observed. The charge transfer resistance in our equivalent circuit is attributed to both electron trapping and detrapping to and from the surface states. ${ }^{9}$ We believe that the increased performance of the Tadoped hematite can be attributed to decreased recombination, enabled by the higher conductivity of the material more efficiently extracting electrons away from the photoanode.

The chemical nature of the surface states observed under illumination with EIS for hematite photoanodes has been convincingly attributed to the formation of a high valence $\mathrm{Fe}(\mathrm{IV})=\mathrm{O}$ species by the Hamann group. ${ }^{25,45,46}$ They showed that the formation of this oxidised surface species resulted in the formation of a new absorption band centered at $572 \mathrm{~nm}$ when the photoanode was kept under anodic bias voltages. ${ }^{25}$ Transient absorption spectroscopy (TAS) performed under operating
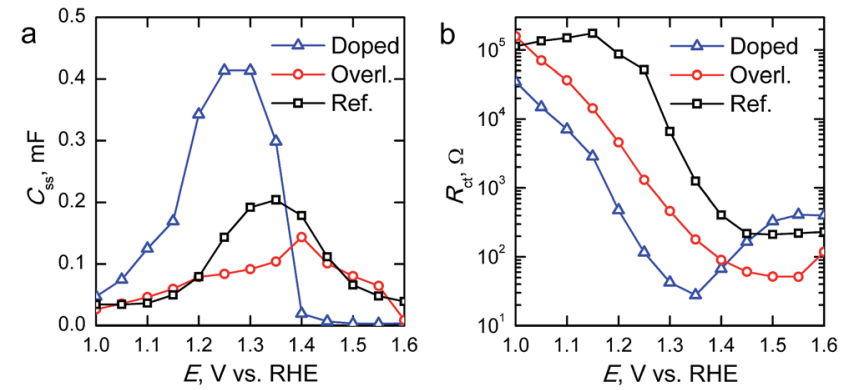

Fig. 7 (a) Surface state capacitances $\left(C_{\text {ss }}\right)$ and (b) charge transfer resistances $\left(R_{\mathrm{ct}}\right)$ of the hematite photoanodes determined under 1 sun AM1.5G illumination. 
conditions has been used to show ultrafast sub-picosecond electron trapping into a surface species on the hematite surface, with the trapping extending well into the millisecond timescale. ${ }^{47,48}$ This trapping is observed as a bleaching (i.e. reduced absorption compared to the ground state) at $575 \mathrm{~nm}$ when an anodic bias voltage is applied. We believe that this bleaching occurs when a photogenerated electron is trapped into the surface state seen in the EIS measurements. We have illustrated earlier that an increase in this electron trapping results in the formation of an increased amount of long-lived holes in the hematite surface, resulting in increased photoelectrochemical performance. ${ }^{48}$ Thus, we performed submillisecond to second timescale TAS measurements under operating conditions to elucidate on the differences in charge carrier dynamics and electron trapping between our photoanodes.

The transient absorption decays of the hematite photoanodes at $575 \mathrm{~nm}$ at selected bias voltages are presented in Fig. 8 and the corresponding biexponential decay component spectra at $1.60 \mathrm{~V} v s$. RHE are presented in Fig. S3. $\dagger$ The decays for the reference hematite photoanode have two components: a negative bleach that disappears completely within $100 \mathrm{~ms}$ and a positive transient absorption on longer timescales that is only visible at bias voltages above the photocurrent onset potential. The lifetimes of the components at $1.60 \mathrm{~V} v s$. RHE are $6.4 \mathrm{~ms}$ and $405 \mathrm{~ms}$ for the bleach and positive signals, respectively. The fast bleach is attributed to prolonged electron trapping into the surface states that we are able to observe with EIS, whereas the positive transient absorption is attributed to long-lived holes in the hematite surface that partake in the water oxidation reaction. ${ }^{47,48}$ The bleach is a localised band with a minimum at $575 \mathrm{~nm}$, whereas the positive transient absorption is observed in the whole monitored region. The transient absorption decays and decay component spectra of the reference hematite are a good match to those we have published earlier for $\mathrm{TiO}_{2}$-doped hematite photoanodes, even though the electron trapping lifetime of our reference hematite is longer than for the $\mathrm{TiO}_{2}$-doped samples (6.4 ms vs. $0.3 \mathrm{~ms}$, respectively). ${ }^{48}$ We attribute this difference to the faster electron extraction dynamics from the surface states due to the preferable band alignment of the $\mathrm{TiO}_{2}{ }^{-}$ doped material. ${ }^{49}$ However, the water oxidation dynamics are practically identical (exponential lifetimes $405 \mathrm{~ms}$ and $400 \mathrm{~ms}$ ).

In this timescale the transient absorption amplitude for the $\mathrm{Ta}_{2} \mathrm{O}_{5}$-overlayer hematite sample is zero at potentials below the photocurrent onset voltage and increases with higher voltages, indicating that all of the transient absorption signal observed originates from long-lived carriers within the depletion region. No negative transient absorption is observed. However, there is a slight dip still visible at $575 \mathrm{~nm}$ in the decay component spectra shown in Fig. S3. $\dagger$ This is further evidence that the bleach component observed for the reference hematite sample originates from trapping of photogenerated electrons into the surface states that we observe with EIS. Since the $\mathrm{Ta}_{2} \mathrm{O}_{5}$-overlayer passivates these surface states, they are not oxidised due to increased anodic bias voltage, and thus cannot act as trap states for electrons. The transient absorption kinetics are also different when compared to the reference hematite sample. The transient absorption decay at $575 \mathrm{~nm}$ is almost an order of magnitude faster, with bi-exponential fit resulting in lifetimes of 3.0 and $65 \mathrm{~ms}$ for the fast and slow decay components, respectively. This indicates that water oxidation occurs faster on the passivated hematite surface. The water photo-oxidation kinetics on hematite surfaces are known to be sluggish. ${ }^{50}$ The hole lifetime in nanostructured $\mathrm{TiO}_{2}$ thin films was measured under similar conditions to be $\sim 50 \mathrm{~ms}$ by Cowan et al.,$^{50}$ which is close to the lifetime of our $\mathrm{Ta}_{2} \mathrm{O}_{5}$-overlayer hematite. Thus, in addition to the reduced recombination due to the surface state passivation, some of the improved photoelectrochemical performance of the $\mathrm{Ta}_{2} \mathrm{O}_{5}$-overlayer hematite films may be due to a photocatalytically more favourable surface with a smaller activation energy barrier. ${ }^{50}$ Tantalum, as a non-3d high valency metal, may improve the catalytic activity via modulating the oxygen evolution reaction energetics in $3 \mathrm{~d}$ metal oxides, such as hematite. ${ }^{51}$

The fast component in the transient absorption decays of the Ta-doped hematite is the fastest of the samples with a lifetime of $1.4 \mathrm{~ms}$ and smallest in amplitude. This is indicative of reduced recombination of the long-lived surface states. ${ }^{48}$ Furthermore, the longer component assigned to the reaction of long-lived surface holes with a lifetime of $95 \mathrm{~ms}$ is four times
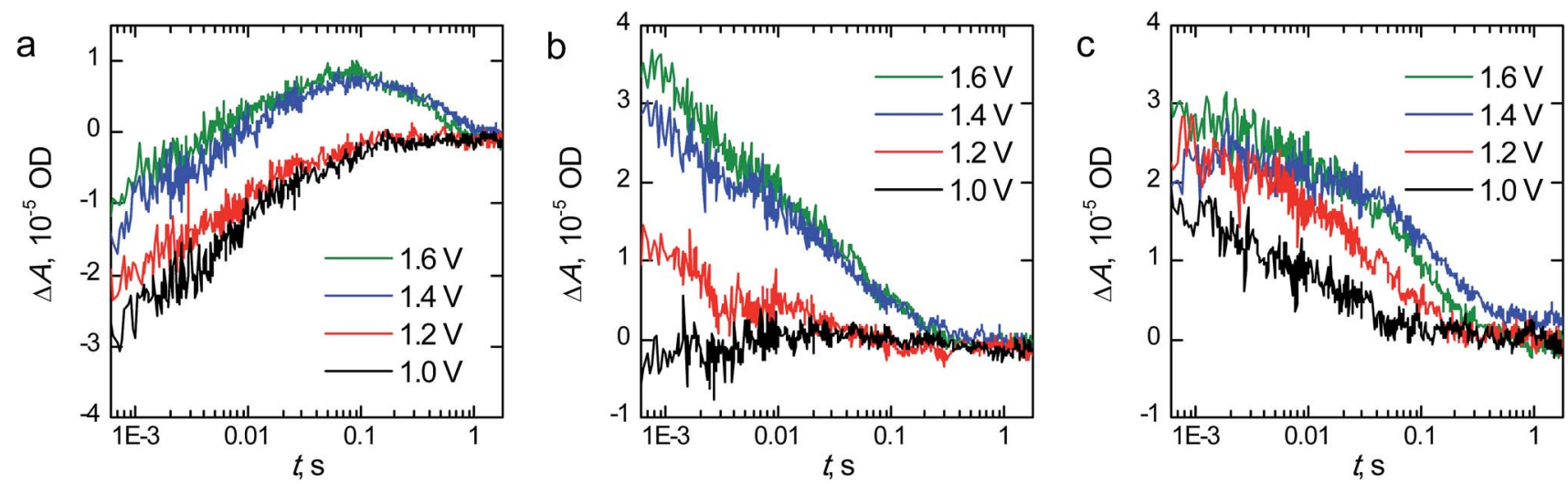

Fig. 8 Sub-millisecond to second timescale transient absorption decays at $575 \mathrm{~nm}$ of (a) reference, (b) $\mathrm{Ta}_{2} \mathrm{O}_{5}$-overlayer, and (c) Ta-doped hematite photoanodes held at selected bias voltages vs. RHE. $\Delta A$ is the change in absorbance in dimensionless optical density (OD) units. 
faster than for the reference hematite sample. We attribute this to the surface of the material having enough tantalum to influence the water photo-oxidation kinetics, making the reaction faster as was observed for the $\mathrm{Ta}_{2} \mathrm{O}_{5}$-overlayer hematite. This is further supported by the similarities between the decay component spectra between the Ta-doped and $\mathrm{Ta}_{2} \mathrm{O}_{5}$-overlayer hematite. The Ta-doped hematite exhibits a positive transient absorption even at $1.0 \mathrm{~V} v s$. RHE. This is presumably due to the observed anodic shift both in the photocurrent and surface state capacitance leading to accumulation of surface holes at lower potentials. The decay at $1.0 \mathrm{~V}$ vs. RHE does not have a long-lived component, indicating that the long-lived holes decay rapidly due to electron back recombination. ${ }^{52}$ As a conclusion, TAS measurements under operating conditions indicate the reason to the increased photoelectrochemical performance of the Ta-doped hematite to be due to reduced recombination and charge trapping to defect states.

Although the Ta-doped hematite does have a dip in the transient absorption centered around $575 \mathrm{~nm}$ that is very similar to the surface passivated $\mathrm{Ta}_{2} \mathrm{O}_{5}$-overlayer hematite (see Fig. S3†), the EIS measurements show that it has the largest surface state capacitance. We would expect that the bleaching in TAS would be more pronounced with samples where the surface state capacitance is larger if the electron trapping occurs to the same surface states that are observed with EIS. From our results it seems that this is not the case here. However, it is also possible that the higher conductivity of the Ta-doped hematite also increases the rate of photoelectron detrapping. We have previously shown that electron trapping in hematite occurs already in the sub-nanosecond timescale. ${ }^{48}$ Forster et al. showed that an $\mathrm{Al}_{2} \mathrm{O}_{3}$-overlayer significantly increased the rate of photoelectron detrapping in oxygen deficient hematite photoanodes, ${ }^{27}$ although the same group did not observe significant changes in the detrapping rate with $\mathrm{Ta}_{2} \mathrm{O}_{5}$-overlayered hematite. ${ }^{36}$ Thus, it is possible that the limited time-scale of our measurement system may miss the trapping in the Ta-doped hematite.

We next turn to transient absorption spectroscopy in the subpicosecond to nanosecond timescale to probe the ultrafast charge carrier relaxation and recombination processes. The transient absorption spectra are presented as contour plots in Fig. 9. The normalized transient absorption spectra at selected delay times are shown in Fig. $\mathrm{S} 4 \uparrow$ and the transient absorption decays at wavelengths with the largest transient absorption and bleach signals are shown in Fig. S5.† On this timescale the transient absorption signal is composed of a superposition of the absorptions of photogenerated electrons, holes, and the ground state bleach. All three samples exhibit a strong positive transient absorption band centered around $585 \mathrm{~nm}$, with a diffuse low energy tail and a sharp high energy edge around the band gap energy. This transient absorption has previously been assigned to originate dominantly from the absorption of photoexcited electrons. ${ }^{53}$ However, recent investigations of hematite photoanodes under applied bias voltages have revealed a positive absorption at the same wavelength that is formed due to a high valence iron-oxo species, as discussed earlier. Furthermore, our previous results show that ultrafast electron trapping into oxidized surface states inverts the positive absorption $\sim 580 \mathrm{~nm}$ into a bleaching signal that we can observe still in the millisecond timescale in Fig. $8 .^{48}$ Thus, we conclude that the main component of the positive transient absorption is due to photogenerated holes in hematite. ${ }^{54}$ All three samples also exhibit a transient bleach signal below $550 \mathrm{~nm}$, attributed to the bleaching of the ground state absorption due to mostly photogenerated electrons, with a secondary weak positive transient absorption band that is split around $500 \mathrm{~nm}$. However, it seems that the bleach intensity grows in the order of reference, $\mathrm{Ta}_{2} \mathrm{O}_{5}$-overlayer, and $\mathrm{Ta}$ doped hematite. The major differences between the samples in the transient absorption spectra lie in the range between 450$550 \mathrm{~nm}$. The transient absorption decays at selected wavelengths from this region are presented in Fig. 10. The four exponential decay component spectra of the different lifetime components are shown in Fig. S6. $\dagger$

We will first consider the ultrafast dynamics in the reference hematite photoanode in Fig. 10a. The initial relaxation of hot electrons takes place with a lifetime of $0.21 \mathrm{ps},{ }^{55,56}$ with the decay amplitude amounting to approximately $50 \%$ of the transient absorption immediately after excitation (see Fig. S5 and S6†). This initial decay is mainly observed at wavelengths with positive transient absorption above $570 \mathrm{~nm}$, although a small bleach recovery is observed at wavelengths below $450 \mathrm{~nm}$ with this lifetime. The fast relaxation is presumably due to the formation of polarons in the hematite bulk, partially trapping the charge carriers. ${ }^{57}$ The generation of itinerant photoexcited electrons ${ }^{56}$ that are free to travel in the conduction band is most clearly observed in the bleach that is formed with a lifetime of $1.6 \mathrm{ps}$ at $530 \mathrm{~nm}$, with a simultaneous decay observed over most of the spectrum above $500 \mathrm{~nm}$. The free conduction electrons are trapped by midgap states, ${ }^{55}$ which is seen as a reduction in the bleach at 530 and $520 \mathrm{~nm}$ and a formation of a positive transient absorption at 500, 485, and $470 \mathrm{~nm}$ with a lifetime of 46 ps. Interestingly, the $470 \mathrm{~nm}$ signal shows almost zero transient absorption until the formation increases its transient absorption. Finally, the remaining free and midgap trapped electrons decay into the ground state with a lifetime of $2.4 \mathrm{~ns}$.

The ultrafast dynamics of the $\mathrm{Ta}_{2} \mathrm{O}_{5}$-overlayer hematite exhibit a few noted differences when compared with the reference hematite. Only the signal at $500 \mathrm{~nm}$ has a positive transient absorption immediately after excitation, having the same 0.21 ps decay component associated with the relaxation of hot electrons. At 470 and $530 \mathrm{~nm}$ a bleach is formed immediately after excitation, with further bleaching with a lifetime of $2.1 \mathrm{ps}$ assigned to the formation of free conduction band electrons observed at 530, 520, and $485 \mathrm{~nm}$. Thus, the formation of free electrons in the $\mathrm{Ta}_{2} \mathrm{O}_{5}$-overlayer hematite is more rapid and observed over a wider energetic range. The trapping of the conduction band electrons by midgap states occurs with a slower lifetime of $61 \mathrm{ps}$, with nearly identical amplitudes as for the reference hematite. Furthermore, the decay of the free and trapped electrons occurs with a lifetime of $3.4 \mathrm{~ns}$, markedly slower than for the reference hematite. We attribute the faster generation and slower recombination dynamics of electrons to a reduction in surface state assisted recombination. 

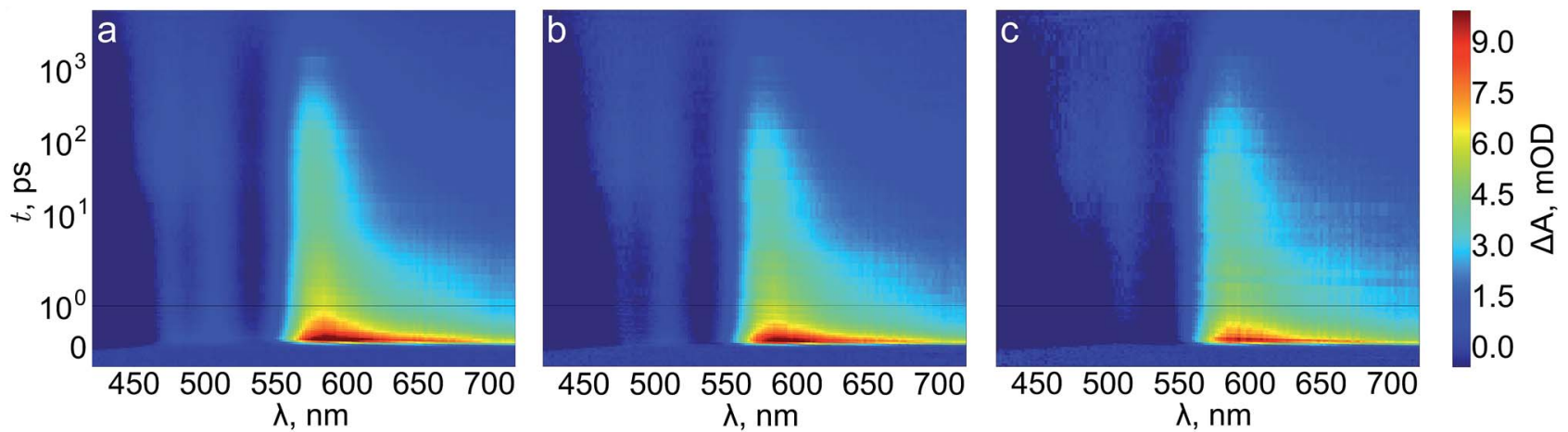

Fig. 9 Transient absorption contour plots of (a) reference, (b) $\mathrm{Ta}_{2} \mathrm{O}_{5}$-overlayer, and (c) Ta-doped hematite photoanodes in air.

However, the transient absorption dynamics for the Tadoped hematite are drastically different from the reference and $\mathrm{Ta}_{2} \mathrm{O}_{5}$-overlayer hematite photoanodes. The bleach at $470 \mathrm{~nm}$ is now three times larger in amplitude than for the $\mathrm{Ta}_{2} \mathrm{O}_{5}$-overlayer hematite, whereas for the reference hematite no bleach was observable at this wavelength. All other wavelengths in the 450-550 $\mathrm{nm}$ range are also bleached immediately after excitation, most clearly visible in the raw and decay component spectra immediately after excitation as shown in Fig. S7. $\dagger$ The bleach recovery has two recovery components with lifetimes of 0.38 and 7.7 ps. We interpret this strong bleach immediately after excitation as indication that even the hot electrons are free to travel in the Ta-doped hematite, instead of being excitonically bound. The $0.38 \mathrm{ps}$ component would then be the thermalization lifetime of the free hot electrons, whereas $7.7 \mathrm{ps}$ is the lifetime of the trapping of these free electrons by the midgap states that form a positive transient absorption at 485 and $500 \mathrm{~nm}$. A small component with a lifetime of 270 ps is observed to reduce the transient absorption at all wavelengths in this range, followed by a decay with a lifetime of $5.9 \mathrm{~ns}$. The observation that free electrons are more freely generated and decay with the longest lifetime in the Ta-doped hematite suggests that the Ta-doping increases the conductivity in the hematite bulk substantially. This conductivity increase alters the ultrafast charge carrier dynamics and separation, leading to noticeable improvements in photoelectrochemical performance.

\section{Conclusions}

We employed atomic layer deposition to prepare $50 \mathrm{~nm}$ thick hematite photoanodes followed by passivating them with a $0.5 \mathrm{~nm}$ thick $\mathrm{Ta}_{2} \mathrm{O}_{5}$-overlayer samples and compared them with samples uniformly doped with same amount of tantalum oxide deposition cycles. The passivating of hematite surface states resulted in a three-fold improvement in photocurrent with the same onset voltage, while electrochemical impedance spectroscopy under visible light irradiation showed a decreased amount of surface states under water splitting conditions. The Ta-doped samples had the same increase in photocurrent along with a $0.15 \mathrm{~V}$ cathodic shift in the onset voltage and decreased resistivity. The surface state capacitance for the Ta-doped sample was double that of the reference photoanode, which implies a larger amount of surface hole accumulation to surface states to which electrons may be trapped into. We utilized transient absorption spectroscopy in the sub-millisecond to second timescale under operating conditions that indicated that electron trapping in both $\mathrm{Ta}_{2} \mathrm{O}_{5}$-passivated and Ta-doped samples was markedly reduced. Ultrafast transient absorption spectroscopy in the sub-picosecond to nanosecond timescale
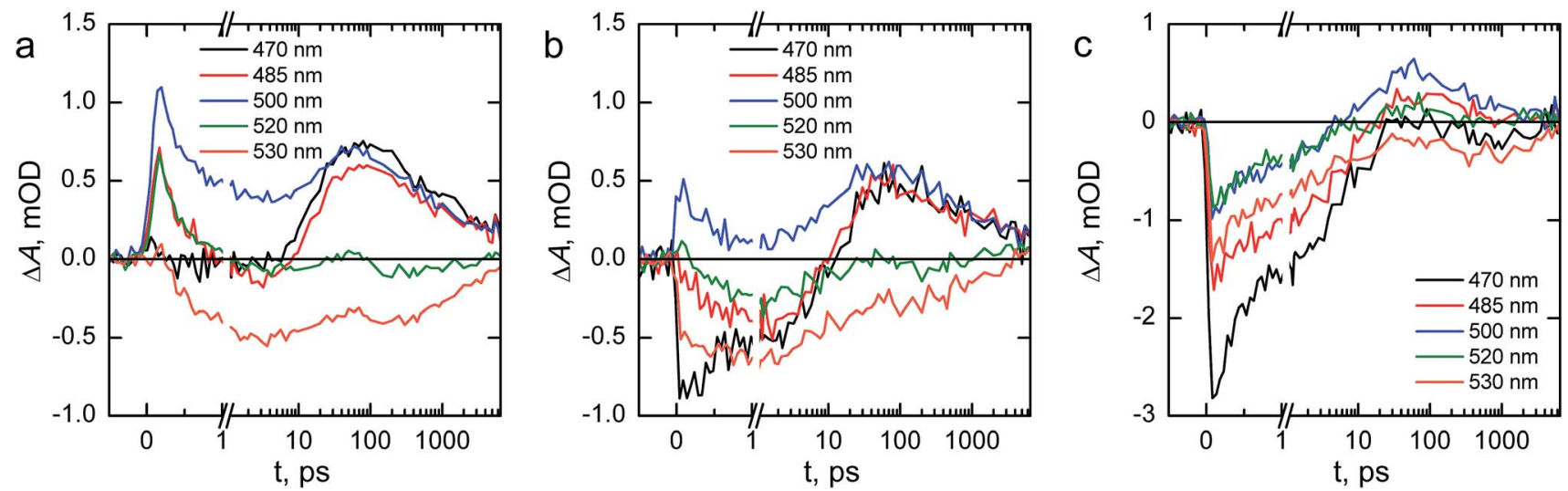

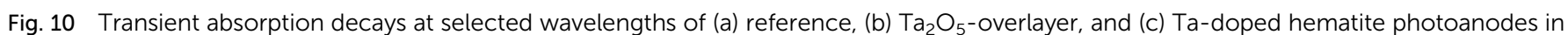
air. The timescale is linear until 1 ps and logarithmic for longer delay times. 
illustrated increased electron mobility with free electrons forming immediately after excitation. Furthermore, the recombination in the Ta-doped hematite photoanode was reduced, resulting in the increased photoelectrochemical performance when compared with the $\mathrm{Ta}_{2} \mathrm{O}_{5}$-overlayer sample. Our results show that the commonly employed passivation strategy does not affect the poor charge carrier dynamics intrinsic to hematite based photoanodes. The large effect on the water splitting performance that electron trapping into surface states has in hematite based photoanodes has only recently been observed. Our Ta-doping strategy results in more efficient electron extraction, solving the electron trapping issue and leading to increased performance over the surface passivation strategy.

\section{Conflicts of interest}

There are no conflicts to declare.

\section{Acknowledgements}

T.-P. R. was supported by the Finnish Cultural Foundation. H. A. was supported by the Jenny and Antti Wihuri Foundation. This work was supported by the Academy of Finland, decision numbers 141481, 286713, and 309920. The research was also supported by the Finnish Centre of Excellence in Atomic Layer Deposition.

\section{References}

1 K. L. Hardee and A. J. Bard, J. Electrochem. Soc., 1976, 123, 1024-1026.

2 A. Murphy, P. Barnes, L. Randeniya, I. Plumb, I. Grey, M. Horne and J. Glasscock, Int. J. Hydrogen Energy, 2006, 31, 1999-2017.

3 K. Sivula, F. Le Formal and M. Grätzel, ChemSusChem, 2011, 4, 432-449.

4 K. Sivula, J. Phys. Chem. Lett., 2013, 4, 1624-1633.

5 R. K. Quinn, R. Nasby and R. Baughman, Mater. Res. Bull., 1976, 11, 1011-1017.

6 J. H. Kennedy and K. W. Frese, J. Electrochem. Soc., 1978, 125, 709-714.

7 M. P. Dare-Edwards, J. B. Goodenough, A. Hamnett and P. R. Trevellick, J. Chem. Soc., Faraday Trans. 1, 1983, 79, 2027-2041.

8 K. Itoh and J. O. Bockris, J. Appl. Phys., 1984, 56, 874-876.

9 L. Bertoluzzi, P. Lopez-Varo, J. A. J. Tejada and J. Bisquert, J. Mater. Chem. A, 2016, 4, 2873-2879.

10 A. Hellman and R. G. Pala, J. Phys. Chem. C, 2011, 115, 12901-12907.

11 B. Iandolo and A. Hellman, Angew. Chem., Int. Ed., 2014, 126, 13622-13626.

12 O. Zandi and T. W. Hamann, J. Phys. Chem. Lett., 2014, 5, 1522-1526.

13 Y. Yang, M. Forster, Y. Ling, G. Wang, T. Zhai, Y. Tong, A. J. Cowan and Y. Li, Angew. Chem., Int. Ed., 2016, 55, 3403-3407.
14 T. Hisatomi, F. Le Formal, M. Cornuz, J. Brillet, N. Tétreault, K. Sivula and M. Grätzel, Energy Environ. Sci., 2011, 4, 25122515.

15 M. Barroso, C. A. Mesa, S. R. Pendlebury, A. J. Cowan, T. Hisatomi, K. Sivula, M. Grätzel, D. R. Klug and J. R. Durrant, Proc. Natl. Acad. Sci. U. S. A., 2012, 109, 15640-15645.

16 C. Du, M. Zhang, J.-W. Jang, Y. Liu, G.-Y. Liu and D. Wang, J. Phys. Chem. C, 2014, 118, 17054-17059.

17 L. Steier, I. Herraiz-Cardona, S. Gimenez, F. FabregatSantiago, J. Bisquert, S. D. Tilley and M. Grätzel, Adv. Funct. Mater., 2014, 24, 7681-7688.

18 R. Liu, Z. Zheng, J. Spurgeon and X. Yang, Energy Environ. Sci., 2014, 7, 2504-2517.

19 T. Wang, Z. Luo, C. Li and J. Gong, Chem. Soc. Rev., 2014, 43, 7469-7484.

20 O. Zandi, B. M. Klahr and T. W. Hamann, Energy Environ. Sci., 2013, 6, 634-642.

21 X. Yang, R. Liu, C. Du, P. Dai, Z. Zheng and D. Wang, ACS Appl. Mater. Interfaces, 2014, 6, 12005-12011.

22 X. Li, P. S. Bassi, P. P. Boix, Y. Fang and L. H. Wong, ACS Appl. Mater. Interfaces, 2015, 7, 16960-16966.

23 F. Le Formal, N. Tétreault, M. Cornuz, T. Moehl, M. Grätzel and K. Sivula, Chem. Sci., 2011, 2, 737-743.

24 F. Le Formal, K. Sivula and M. Grätzel, J. Phys. Chem. C, 2012, 116, 26707-26720.

25 B. Klahr and T. Hamann, J. Phys. Chem. C, 2014, 118, 1039310399.

26 M. G. Ahmed, I. E. Kretschmer, T. A. Kandiel, A. Y. Ahmed, F. A. Rashwan and D. W. Bahnemann, ACS Appl. Mater. Interfaces, 2015, 7, 24053-24062.

27 M. Forster, R. J. Potter, Y. Ling, Y. Yang, D. R. Klug, Y. Li and A. J. Cowan, Chem. Sci., 2015, 6, 4009-4016.

28 W.-J. Chun, A. Ishikawa, H. Fujisawa, T. Takata, J. N. Kondo, M. Hara, M. Kawai, Y. Matsumoto and K. Domen, J. Phys. Chem. B, 2003, 107, 1798-1803.

29 C. Chaneliere, J. Autran, R. Devine and B. Balland, Mater. Sci. Eng., $R, 1998,22,269-322$.

30 P. Zhang, J. Zhang and J. Gong, Chem. Soc. Rev., 2014, 43, 4395-4422.

31 V. Aroutiounian, V. Arakelyan, G. Shahnazaryan, G. Stepanyan, J. A. Turner and O. Khaselev, Int. J. Hydrogen Energy, 2002, 27, 33-38.

32 C. Jorand-Sartoretti, B. D. Alexander, R. Solarska, I. A. Rutkowska, J. Augustynski and R. Cerny, J. Phys. Chem. B, 2005, 109, 13685-13692.

33 Y. Fu, C.-L. Dong, Z. Zhou, W.-Y. Lee, J. Chen, P. Guo, L. Zhao and S. Shen, Phys. Chem. Chem. Phys., 2016, 18, 3846-3853.

34 X. Zhang, H. Li, S. Wang, F.-R. F. Fan and A. J. Bard, J. Phys. Chem. C, 2014, 118, 16842-16850.

35 C. Li, T. Wang, Z. Luo, D. Zhang and J. Gong, Chem. Commun., 2015, 51, 7290-7293.

36 M. Forster, R. J. Potter, Y. Yang, Y. Li and A. J. Cowan, ChemPhotoChem, 2018, 2, 183-189.

37 J. A. Klug, N. G. Becker, S. C. Riha, A. B. Martinson, J. W. Elam, M. J. Pellin and T. Proslier, J. Mater. Chem. A, 2013, 1, 11607-11613. 
38 T. Suntola, Thin Solid Films, 1992, 216, 84-89.

39 K. Kukli, M. Ritala and M. Leskelä, J. Electrochem. Soc., 1995, 142, 1670-1675.

40 K. Lahtonen, M. Lampimäki, P. Jussila, M. Hirsimäki and M. Valden, Rev. Sci. Instrum., 2006, 77, 083901.

41 J. H. Scofield, J. Electron Spectrosc. Relat. Phenom., 1976, 8, 129-137.

42 J. Jokinen, J. Keinonen, P. Tikkanen, A. Kuronen, T. Ahlgren and K. Nordlund, Nucl. Instrum. Methods Phys. Res., Sect. B, 1996, 119, 533-542.

43 M. C. Biesinger, B. P. Payne, A. P. Grosvenor, L. W. Lau, A. R. Gerson and R. S. C. Smart, Appl. Surf. Sci., 2011, 257, 2717-2730.

44 B. Klahr, S. Gimenez, F. Fabregat-Santiago, T. Hamann and J. Bisquert, J. Am. Chem. Soc., 2012, 134, 4294-4302.

45 B. Klahr, S. Gimenez, F. Fabregat-Santiago, J. Bisquert and T. W. Hamann, Energy Environ. Sci., 2012, 5, 7626-7636.

46 O. Zandi and T. W. Hamann, Nat. Chem., 2016, 8, 778-783.

47 S. R. Pendlebury, X. Wang, F. Le Formal, M. Cornuz, A. Kafizas, S. D. Tilley, M. Grätzel and J. R. Durrant, J. Am. Chem. Soc., 2014, 136, 9854-9857.

48 T.-P. Ruoko, K. Kaunisto, M. Bärtsch, J. Pohjola, A. Hiltunen, M. Niederberger, N. V. Tkachenko and H. Lemmetyinen, J. Phys. Chem. Lett., 2015, 6, 2859-2864.
49 D. Monllor-Satoca, M. Bärtsch, C. Fàbrega, A. Genç, S. Reinhard, T. Andreu, J. Arbiol, M. Niederberger and J. R. Morante, Energy Environ. Sci., 2015, 8, 3242-3254.

50 A. J. Cowan, C. J. Barnett, S. R. Pendlebury, M. Barroso, K. Sivula, M. Grätzel, J. R. Durrant and D. R. Klug, J. Am. Chem. Soc., 2011, 133, 10134-10140.

51 B. Zhang, X. Zheng, O. Voznyy, R. Comin, M. Bajdich, M. García-Melchor, L. Han, J. Xu, M. Liu, L. Zheng, F. P. García de Arquer, C. T. Dinh, F. Fan, M. Yuan, E. Yassitepe, N. Chen, T. Regier, P. Liu, Y. Li, P. De Luna, A. Janmohamed, H. L. Xin, H. Yang, A. Vojvodic and E. H. Sargent, Science, 2016, 352, 333-337.

52 F. Le Formal, S. R. Pendlebury, M. Cornuz, S. D. Tilley, M. Grätzel and J. R. Durrant, J. Am. Chem. Soc., 2014, 136, 2564-2574.

53 S. Sorenson, E. Driscoll, S. Haghighat and J. M. Dawlaty, J. Phys. Chem. C, 2014, 118, 23621-23626.

54 A. Hiltunen, T.-P. Ruoko, T. Iivonen, K. Lahtonen, H. AliLöytty, E. Sarlin, M. Valden, M. Leskelä and N. Tkachenko, Sustainable Energy Fuels, 2018, 2, 2124-2130.

55 A. G. Joly, J. R. Williams, S. A. Chambers, G. Xiong, W. P. Hess and D. M. Laman, J. Appl. Phys., 2006, 99, 053521.

56 L. Fu, Z. Wu, X. Ai, J. Zhang, Y. Nie, S. Xie, G. Yang and B. Zou, J. Chem. Phys., 2004, 120, 3406-3413.

57 J. Husek, A. Cirri, S. Biswas and L. R. Baker, Chem. Sci., 2017, 8, 8170-8178. 